\title{
Science behind AI: the Evolution of Trend, Mobility, and Collaboration
}

\author{
Sha Yuan ${ }^{1,3 \dagger} \cdot$ Zhou Shao ${ }^{2,1 \dagger} \cdot$ Xingxing \\ Wei $^{4}$. Jie Tang ${ }^{*}$. Wendy Hall ${ }^{5}$. \\ Yongli Wang ${ }^{2}$ - Ying Wang ${ }^{6}$ - Ye Wang ${ }^{6}$
}

Received: date / Accepted: date

\begin{abstract}
In this era of interdisciplinary science, many scientific achievements, such as artificial intelligence (AI), have brought dramatic revolutions to human society. The increasing availability of digital data on scholarly outputs offers unprecedented opportunities to explore science of science (SciSci). Despite many significant works have been done on SciSci, substantial disciplinary differences in different domains make some insights inadequate within particular fields. One thing standing out is that knowledge concerning the science behind AI is sorely lacking. In this work, we study the evolution of AI from three dimensions, including the evolution of trend, mobility, and collaboration. We find that the AI research hotspots have shifted from theory to application. The USA, which has the largest number of distinguished AI scientists, appeals most to the global AI talents. The brain drain problem of AI scientists is increasingly serious in developing countries. The ties among the AI elites are highly clustered in the collaboration network. Overall, our work aims to serve as a starter and support the development of AI exploring in a visionary way. The related demos are available online in AMiner ${ }^{1,2}$.
\end{abstract}

Keywords Artificial Intelligence · Science of Science · Trend · Career Trajectory · Collaboration

\footnotetext{
$\dagger$ Sha Yuan and Zhou Shao are co-first authors of the article. They contribute equally to this article.

* Jie Tang is the corresponding author. E-mail: jietang@tsinghua.edu.cn

1 Department of Computer Science and Technology, Tsinghua University

2 Nanjing University of Science and Technology

3 Beijing Academy of Artificial Intelligence

4 Beihang University

5 University of Southampton

6 Exchange, Development \& Service Center for Science Technology Talents, the Ministry of Science and Technology of China

1 https://www.aminer.cn/ai10

2 https://trend.aminer.org
} 


\section{Introduction}

In this era of interdisciplinary science, many scientific achievements, especially in artificial intelligence (AI), have brought dramatic revolutions to human society. With the rapid development and application of AI, it has taken the focus attentions from both industry and academia. The AI field is a broad discipline that draws upon computer science, mathematics, artificial psychology, linguistics, and many others. Although AI is difficult to define precisely, a machine is considered to have intelligence if it passes the Turing test. Thus, artificial intelligence usually refers to an artificial machine or program that simulates human thinking. It exhibits similar intelligent behavior to the human mind. There is no doubt that progress in AI has accelerated the information construction of science and technology. However, the way that AI advances itself is much less well-understood.

The increasing availability of digital data on scholarly outcomes, from the research paper, citation, mobility of scientists to their collaboration relationship, offers unprecedented opportunities to explore science of science (SciSci) [8]. Based on big data based empirical analysis, SciSci provides a quantitative understanding of the interactions among scientific researchers across diverse geographic and temporal scales. It captures the key point in the development process of science. For instance, the digitization of scientific publications itself is driven by the development of science, and now it provides a way to explore the development of scientific research in return. With a deeper understanding of the factors that drive successful science, SciSci generates insight into the current scientific innovations.

Many significant works on SciSci have been done to explore patterns characterizing the structure and evolution of science. The recent development in the SciSci field has been reviewed [8]. The evolution of science over a long time frame has also been unveiled [7]. These work focus on all fields of science in an aggregated way, and the findings are integrated from multiple disciplines. Despite the discovery of universals across science, substantial disciplinary differences in different domains make some insights inadequate within particular fields. Domain-specific SciSci studies are required due to the difference among disciplines. In recent work, the analysis of physical papers spanning more than 100 years, which are collected from Web of Science, reveals the rapid growth and increasing multidisciplinarity of physics [23].

Notwithstanding the driving force of AI in societal development, the scientific knowledge behind AI is sorely lacking. The development of SciSci and big scholarly data offer an unprecedented opportunity to explore the science behind AI. Most of the existing works on SciSci are carried out on digitalized papers collected from the network. However, a very recent study [15] draws out the increasing concentration of scientific prize within a proportionately small and tightly interconnected elite. These relatively constrained number of scientific elites push the boundaries of science. It means that a small number of elite scientists promote the development of science. Based on this finding, we 
explore the science behind AI using the data set of distinguished AI scholars in AMiner [28]. The details of the data set will be described in Section 2.

In this work, we study the evolution of AI from three respects. First, we explore the evolution process of the popular sub-domains in AI. Second, we investigate the mobility patterns of AI scientists at both the national and individual levels. Finally, we characterize the collaboration network between the distinguished AI scholars. Our study aims to explore the evolution patterns of AI. The main findings are listed as follows.

1). AI scientists are more inclined to work on mathematical and theoretical problems in the early development stage of AI. And then they prefer to do application-oriented research in the subsequent period. In the 21st century, the productivity of AI-related papers significantly increase. In this period, application-oriented researches get more attention from AI scientists.

2). Among the distinguished AI scholars in the world, the proportion of American scientists is $46.06 \%$. Chinese, German, British, and Canadian AI scientists account for $9.21 \%, 6.61 \%, 5.37 \%$, and $3.84 \%$, respectively. The global migration statistics of AI scientists show that the migration rate of AI scientists in these five countries is also higher than others in the world. USA, Singapore, and Australia, these three countries are the most popular choice for the AI scientists who plan to emigrate. The difference value of China is the largest negative value. The brain drain of leading AI scientists is a continuing problem in developing countries.

3). We analyze the career trajectory of the distinguished AI scholars in different periods. The lines of career trajectory are sparse in the map in the period of $1970-1988$ and then increase obviously in the period of $1989-1998$. In this period, the AI scientists are mainly distributed in Northern America and Europe. In the new century, the scale of career trajectory lines takes a quantum leap. The lines of career trajectory on the map also increase a lot in the latest ten years. But the increase is not as obvious as in the last decade.

4). In the collaboration network of distinguished AI scientists, 95.11\% scholars are interconnected with each other, and most of them have more than 10 collaborators who are also distinguished AI scientists. In general, the collaboration ties among the AI elites are highly clustered.

5). The distinguished AI scholars are in high mobility, and they get a new job position in 2 to 3 years. They are more productive in the new job position. The publication number of every scholar will increase 2.55 in average after a job-hopping. The number of collaborators remains essentially unchanged, no matter whether the job position of AI scientists changes or not. Most of the cooperative relations among distinguished AI scholars are still maintained even when their job positions have changed. At the same time, the number of new collaborators will increase 2 after a job-hopping.

Overall, our findings demonstrate that AI research hotspots have shifted from theory to application. Mobility is beneficial for creativity. The brain drain of leading AI scientists is still a serious problem in developing countries. There is no doubt that the USA is the AI leader in the world. It is not only the country with the largest number of distinguished AI scientists but also 
the most attractive developed country for global AI talents. The distinguished AI scientist in the world works closely with each other. The collaboration ties among these AI elites are highly clustered. The conclusion that a relatively small group of scientific elites with highly ties push the boundaries of science [15] also holds true in the field of AI.

\section{Data: AMiner}

AMiner $^{3}$ is a free online academic search and mining system, with the emphasis to offer approaches to gain a deeper understanding of the scientific literature data [28]. The system has been in operation since 2006 and has attracted more than 8,000, 000 independent IP accesses from over 220 countries/regions. AMiner automatically extracts researchers' profiles from the Web and integrates the publication data from online databases such as DBLP, ACM Digital Library, CiteSeer, and so on. Currently, there are more than 231, 832, 378 publications and 127, 513, 531 researchers included in AMiner. AMiner has become a strategic partner of Microsoft Academic Search and the official content provider of Sogou3 Scholar. AMiner provides a series of influential applications, including Hotspot Trend ${ }^{4}$, Distinguished AI Scholars ${ }^{5}$, Career Trajectory ${ }^{6}$, and so on.

Table 1: Global Distribution of AI Experts

\begin{tabular}{cccc}
\hline Country & AI scholar & H-index 30 & H-index 60 \\
\hline USA & $39.71 \%$ & $54.13 \%$ & $68 \%$ \\
CHN & $14.77 \%$ & $10.94 \%$ & $6.36 \%$ \\
GBR & $6.30 \%$ & $6.21 \%$ & $5.18 \%$ \\
CAN & $3.29 \%$ & $3.97 \%$ & $3.66 \%$ \\
ISR & $2.12 \%$ & $1.86 \%$ & $1.19 \%$ \\
DEU & $2.92 \%$ & $2.27 \%$ & $1.65 \%$ \\
AUS & $2.58 \%$ & $2.01 \%$ & $1.41 \%$ \\
NLD & $2.57 \%$ & $2.35 \%$ & $1.41 \%$ \\
CHE & $1.15 \%$ & $1.33 \%$ & $1.40 \%$ \\
ITA & $3.69 \%$ & $2.41 \%$ & $1.18 \%$ \\
\hline
\end{tabular}

In particular, this paper uses 3-letter country abbreviation (ISO-3166-1 ALPHA-3) as the code for the country.

\footnotetext{
3 https://www.aminer.cn

4 https://trend.aminer.cn

5 https://www.aminer.cn/ai10

6 https://traj.aminer.cn/trajectory-index
} 


\subsection{AI Scholars in AMiner}

We statistically analyze the AI academic data in AMiner. There are 18107 AI experts in the world, in which:

- the global AI Experts are distributed in 67 different countries and regions around the world.

- the number of AI experts, whose H-Index is greater than or equal to 30, is 4918. This number accounts for $27.16 \%$ of the total AI experts. These AI experts are distributed in 43 different countries and regions worldwide.

- the number of AI experts, whose H-Index is greater than or equal to 60, is 742 . The number accounts for $4.10 \%$ of the total AI experts. These AI experts are distributed in 25 different countries and regions worldwide.

- there are 17231 males and 876 females. The males account for $95.16 \%$ of the total AI experts.

The proportion of AI experts in each country or region is illustrated in Table. 1. The top three countries with the largest number of AI scientists are USA, CHN, and GBR.

\subsection{Most Influential AI Scholars}

The AMiner Most Influential Scholar Annual List in Artificial Intelligence (AI) names the world's top-cited research scholars from the fields of AI. The list is conferred in recognition of outstanding technical achievements with lasting contribution and impact to the research community. In 2018, this list aimed to find the most cited AI scholars in the top venues in the latest 10 years. We collect AI papers published in the top venues of their respective subject fields between 2007 and 2017. The authors of the most cited papers among these papers are marked as AI-10 scholars in AMiner. Recipients are automatically determined by a computer algorithm deployed in the AMiner system. Scholars are ranked based on citation counts collected by top-venue publications.

The development of science is largely inspired by previous researches of distinguished scientists. The latest research [15] shows that the scientific prize is more concentrated within a relatively small group of scientific elites, and a relatively constrained number of distinguished scholars push the boundaries of science. And the boundaries of science are pushed by a relatively small group of scientific elites. The experts in the AI-10 expert base are the leading and distinguished AI scientists in the world with no doubt. In this paper, our work aims to understand the evolution of AI through the distinguished AI scientists in the AI-10 expert base, which focuses on three dimensions: trend, mobility, and scientific collaboration. We design several questions in these dimensions to reveal the science behind AI. In specific, along each dimension, we proceed with our studies by answering a set of insightful questions, which is summarized in Table 2. 
Table 2: Summary of Questions Answered from the Data.

\begin{tabular}{|c|c|}
\hline Dimension & Questions \\
\hline Trend & $\begin{array}{l}\text { Q3.1: What are the most popular research fields in AI? } \\
\text { Q3.2: What are the most popular AI fields in different periods? }\end{array}$ \\
\hline Mobility & $\begin{array}{l}\text { Q4.1: What's the global distribution of AI scholars? } \\
\text { Q4.2: What's the migration situation of AI scholars? } \\
\text { Q4.3: What does the AI scientist's career trajectory look like? } \\
\text { Q4.4: How often do AI scientists change their job positions? } \\
\text { Q4.5: What's the relationship between mobility and creativity? }\end{array}$ \\
\hline Collaboration & $\begin{array}{l}\text { Q5.1: What is the distribution of collaboration numbers? } \\
\text { Q5.2: What is the collaboration network between AI scientists? } \\
\text { Q5.3: How does the number of collaborators change? } \\
\text { Q5.4: How many new collaborators have been increased? } \\
\text { Q5.5: How many collaborators will be retained? }\end{array}$ \\
\hline
\end{tabular}

\section{The Evolution of Trend}

Nowadays, AI technology has become an important part of the technology industry. Many challenging application problems are solved by AI and achieved excellent human-like performance. In this section, we present the development trend of AI according to the research work favored by the distinguished AI scientists.

\subsection{The Overall Trend}

Q3.1: What are the most popular research fields in AI?

The latest research results suggest that the development trend of science is leading by distinguished scientists. To answer this question, we focus on the most popular research subjects favored by the distinguished AI scientist to explore the evolution trend of AI.

Figure 1 provides the top 12 popular research keywords from 1970 to 2018, including feature extraction, data mining, computer vision, machine learning, mobile robots, support vector machines, Hidden Markov models, computer science, humanoid robots, real-time, speech recognition, and computational complexity. The list, which is arranged by popularity, contains the research AI keywords both in theory and application. Computer science, which is the father field of artificial intelligence, is also in the AI trend list.

Although the scope of AI is disputed, we can extrapolate from the trend list that data mining, machine learning, computer vision, robots, speech recognition are the most popular subfields of AI. Feature extraction is the keyword that is repeated most often in the papers of the distinguished AI scientists. It is an important step in data preprocessing, which is one of the most significant steps in the learning process of AI. Feature extraction constructs feature from the initial data set to provide useful information for processing. It leads to 


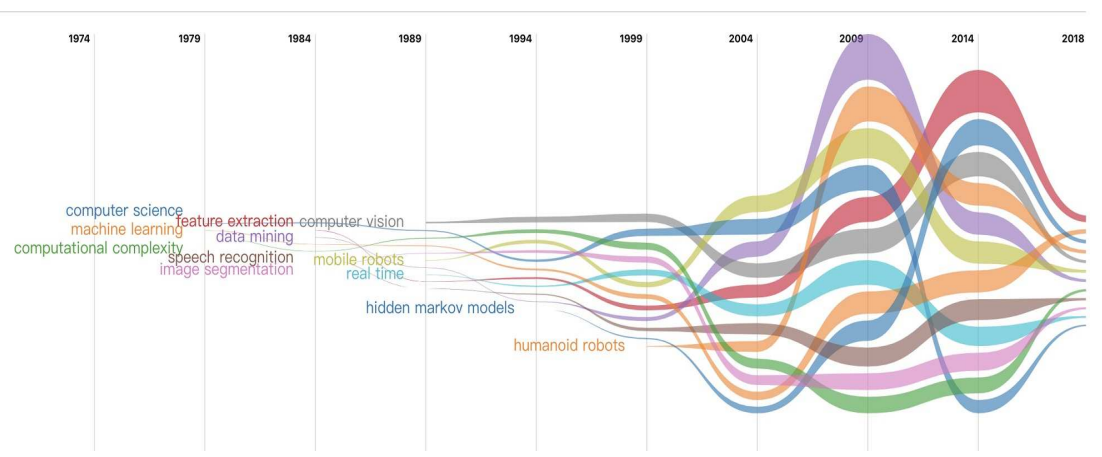

Fig. 1: The Evolution Trend of AI. The most popular research fields in AI are feature extraction, data mining, computer vision, machine learning, mobile robots, support vector machines, hidden markov models, computer science, humanoid robots, real time, speech recognition, and computational complexity.

Table 3: Development Trend in Each Period

\begin{tabular}{llll}
\hline $1970-1988$ & $1989-1998$ & $1999-2008$ & $2009-2018$ \\
\hline artificial intelligence & computer vision & computer science & feature extraction \\
computer science & neural network & data mining & data mining \\
image processing & motion estimation & mobile robots & social network \\
parameter estimation & computational complexity & feature extraction & hidden markov models \\
mathematical model & image segmentation & computer vision & wireless sensor networks \\
linear programming & parameter estimation & real time & computer vision \\
polynomial time & computer science & speech recognition & computational modeling \\
computational complexity & feature extraction & support vector machines & machine learning \\
pattern recognition & mobile robots & machine learning & speech recognition \\
knowledge base & signal processing & humanoid robots & real time \\
maximum likelihood estimation & real time & image segmentation & mobile robots \\
signal processing & pattern recognition & neural network & support vector machines \\
\hline
\end{tabular}

better human interpretation and facilitates subsequent learning and generalization steps. Hidden Markov model and support vector machine are especially known for their successful application in AI. Real-time and low computational complexity are two basic implementation requirements of AI.

\subsection{Trend in Different Periods}

Q3.2: What are the most popular AI fields in different periods?

We list the top 12 popular research keywords of AI in the period of $1970-$ 1988, $1989-1998,1999-2008$, and $2009-2018$ in Table 3. The hot AI research fields in each period are branded with a distinct era mark. Computer science is the father field of artificial intelligence. As one of the most popular AI keywords, it appears in the list in the period of $1970-1988,1989-1998$ and $1999-2008$, but not in the period of $2009-2018$. It may suggest that AI 
has become a new independent discipline, not just the subfield of computer science.

In the period of $1970-1988$, the mathematics and theory related AI researches are most popular, such as parameter estimation, mathematical model, linear programming, polynomial time, computational complexity, and maximum likelihood estimation. In this era, the mathematics and theory related AI researches account for a significant proportion. In the following period of 1989 - 1998, the proportion of mathematics-related AI researches declines. Computer vision related fields (including motion estimation, image segmentation, and so on) catch the eyes of AI scientists in this period. Most of the popular research keywords in the period of $1999-2008$ and $2009-2018$ are also the popular keywords in the total period, such as data mining, feature extraction, computer vision, speech recognition, support vector machine, and so on. It is because that there is a large growth in the number of AI research outcomes for the last 20 years due to the development of new technologies (such as Web technology, big data, cloud computing, and so on) in the new century. In the overall statistic, the popular AI research keywords in these two periods account for a large proportion. In the period of $2009-2018$, social network and wireless sensor network, which are the new application that appeared in recent years, are also listed in table 3 .

The popular research keywords in each period show that the AI researches prefer to explore mathematics and theory related researches in the early period, and applied-oriented researches in the following period. In the twentyfirst century, AI techniques have experienced a resurgence following concurrent advances in theoretical understanding, computing power, and big data. AI techniques have become an essential part of the technology industry, helping to solve many challenging application problems. Artificial intelligence has achieved excellent human-like performance in some domains, such as speech recognition, image recognition, and so on. It is precisely because of outstanding performance. Artificial intelligence sets off an upsurge of AI researches in the scientific community and AI applications in the industry.

\section{Mobility}

The career paths of scientists are not static. Changing institutions is an integral part of academic life. Every publication lists the institution where the work was performed. The career trajectories of AI scientists are reflected in their publication on a fine-scale and in great detail [21]. In this section, we explore the global mobility ${ }^{7}$ of the distinguished AI scientists. Firstly, we statistically analyze the global distribution of these AI scientists. And then, we explore underlying trends in AI scientists' movements from a national perspective. Thirdly, we seek to identify the career trajectory of the distinguished AI scientists in different periods, and each period is measured in ten years. Fi-

\footnotetext{
7 http://static.aminer.org/lab-datasets/trajectory/top10000career-trajectory.mp4
} 


\begin{tabular}{ccc||ccc}
\hline Country & Number & Ratio & Country & Number & Ratio \\
\hline USA & 1080 & $46.06 \%$ & JPN & 48 & $2.05 \%$ \\
CHN & 216 & $9.21 \%$ & ISR & 48 & $2.05 \%$ \\
DEU & 155 & $6.61 \%$ & SGP & 38 & $1.62 \%$ \\
GBR & 126 & $5.37 \%$ & NLD & 37 & $1.58 \%$ \\
CAN & 90 & $3.84 \%$ & IND & 33 & $1.41 \%$ \\
ITA & 78 & $3.33 \%$ & AUT & 32 & $1.36 \%$ \\
FRA & 60 & $2.56 \%$ & ESP & 32 & $1.36 \%$ \\
CHE & 55 & $2.35 \%$ & AUS & 29 & $1.24 \%$ \\
\hline
\end{tabular}

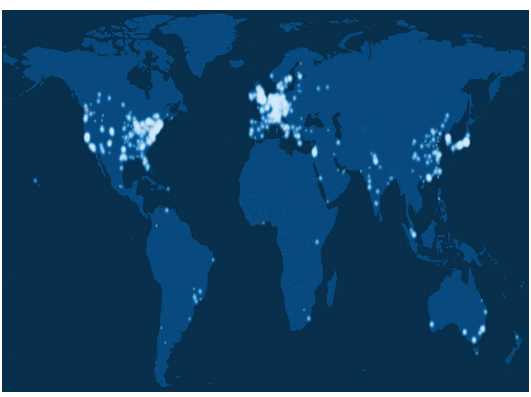

Fig. 2: The number.

Fig. 3: The Global Distribution of AI Experts.

nally, we extract the relationship between mobility and productivity to explore how mobility patterns affect scientific outcomes.

\subsection{Global Distribution}

Q4.1: What's the global distribution of AI researches?

We statistically analyze the AI academic data in AMiner. There are 18107 AI experts in the world, in which the number of distinguished AI experts in the AMiner AI-10 Most Influential Scholars List is 2246, accounting for $12.40 \%$. We explore the global distribution of these distinguished AI experts, and the results are illustrated in Fig. 2 and 3. The proportion of distinguished AI experts in the top 16 countries and regions are listed in Fig. 2. Among the distinguished AI experts in the world, the proportion of American scientists is $46.06 \%$, which is nearly half of the total number of global distinguished AI experts. Being number one, the USA is effectively the AI leader.

Combined with the H-index influence of AI experts with the number distribution, we illustrate the heat distribution of the distinguished AI experts in Fig. 3. It clearly shows that most of the distinguished AI experts are gathered in three regions in the world, including Northern America, Europe, and East Asia. In Northern America, distinguished AI scholars are mainly distributed in the United States and Canada. In Europe, the distinguished AI scholars are distributed in many countries. In East Asia, the distinguished AI scholars are mainly distributed in China, Japan, and Korea. In China, the distinguished AI scholars are mainly distributed in the southeast coastal area, which is the most economically developed area in China.

\subsection{Migration Situation}

Q4.2: What's the migration situation of AI scholars?

Under this question, we seek to identify underlying trends in AI scientists' movements from a national perspective. We track the change of the AI scien- 
Table 4: Migration Statistic of AI Scientists

\begin{tabular}{cccc||cccc}
\hline Country & In & Out & Difference & Country & In & Out & Difference \\
\hline USA & 520 & -473 & +47 & FRA & 33 & -39 & -6 \\
CHN & 121 & -144 & -23 & AUT & 34 & -33 & +1 \\
GBR & 115 & -110 & +5 & JPN & 28 & -28 & 0 \\
DEU & 98 & -108 & -10 & AUS & 31 & -24 & +7 \\
CAN & 62 & -66 & -4 & KOR & 26 & -26 & 0 \\
CHE & 58 & -56 & +2 & SGP & 29 & -21 & +8 \\
IND & 45 & -50 & -5 & MYS & 19 & -20 & -1 \\
ISR & 41 & -51 & -10 & SWE & 17 & -18 & -1 \\
ITA & 35 & -41 & -6 & CZE & 16 & -15 & +1 \\
NLD & 37 & -38 & -1 & IRL & 15 & -14 & +1 \\
\hline
\end{tabular}

tists' serving establishment to explore the migration situation. Nature reports that one in eight of the world's most highly cited scientists from 1981 to 2003 were born in developing countries, but $80 \%$ of them had since moved to developed countries (mostly the United States) [29]. The migration statistic of the distinguished AI scientists listed in Table 4 also shows a similar trend. The number of distinguished AI scientist immigrating to the USA occupies nearly half $(48.15 \%)$ of the total number of distinguished AI scientists in the world. There are 520 distinguished AI scientists move into the USA, and 473 move out. The scale of migrations in the USA is relatively larger than other countries. China and Great Britain are in the second and third place, respectively. But the number of AI scientists moving out of China is more than the number of AI scientists moving in.

As listed in Table 4, the difference values in USA $(+47), \operatorname{SGP}(+8), \operatorname{AUS}(+7)$, GBR $(+5), \operatorname{CHE}(+2), \operatorname{AUT}(+1), \mathrm{ZCE}(+1)$ and IRL $(+1)$ are positive. The finding suggests that most of distinguished AI scientists move into the USA, followed by Singapore and Australia. These three countries are the most popular choice for the distinguished AI scientists who tend to emigrate. The difference value of China is -23 , which is the largest negative value. The brain drain problem of distinguished AI scientists is still serious in China. Although the details of the migration are blurry, it is obvious that the United States has attracted the global AI talents and benefited from the openness to foreign AI scientists. The developing countries, especially China, need to take measures to retain AI talents. From a policy perspective, a developing country should target young people who have studied overseas and try to attract them back, because they are more likely to move. According to the report in Nature [29], those that do are seven times more likely to return homeland between the ages of 35 and 45 than after 50 . 

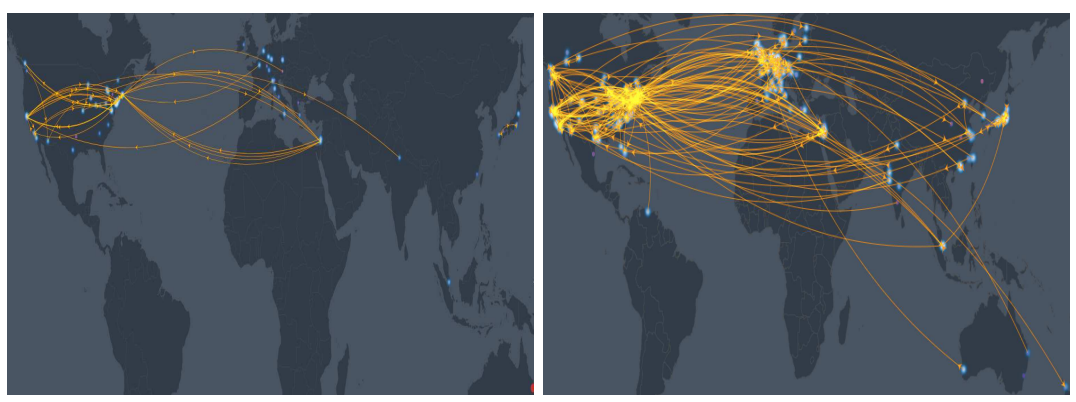

Fig. 4: AI scientists' trajectories of Fig. 5: AI scientists' trajectories of 1970 to 1988.

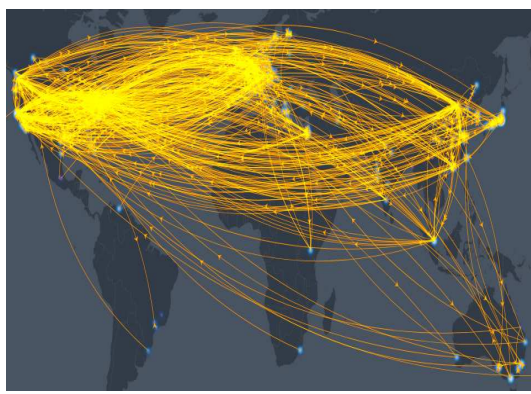
1989 to 1998.

Fig. 6: AI scientists' trajectories of Fig. 7: AI scientists' trajectories of 1999 to 2008

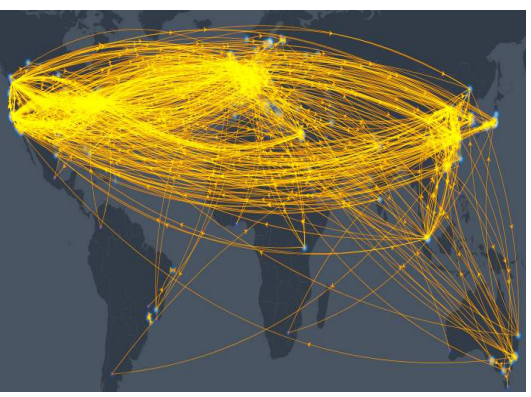
2009 to 2018.

\subsection{Career Trajectory}

In the scholar trajectory system, we extract the name of the institution mentioned in the affiliation string, and leverage Google Map API to map the institution name to its corresponding geographic location. We formulate the problem as a sequential labeling task. An affiliation string is broken into a quadruplet < scholarId, institution, year, geoLocation > using the CRF model, where scholarId is the unique identifier of a researcher, year is the publication year, institution is the extracted institution name from the affiliation string, and geoLocation is the corresponding geographic coordinates. In this subsection, we try to uncover the career trajectory of distinguished AI scholars in different periods.

Q4.3: What does the AI scientist's career trajectory look like?

Individual academic careers of AI scholars unfold in the context of a vast market for artificial intelligence. We analyze the career trajectory of the distinguished AI scientists in the period of 1970 - 1988, 1989-1998, 1999-2008, and $2009-2018$. As shown in Fig. 4, the lines of career trajectory are sparse in the map in the period of $1970-1988$. And then, the career trajectory lines increase obviously in the period of $1989-1998$ as shown in Fig. 5. In this period, the distinguished AI scientists are mainly distributed in Northern 
America and Europe. As shown in Fig. 6, the scale of career trajectory lines takes a quantum leap in the new century. The career trajectories on the map are distributed densely and mainly in Northern America, Europe, and East Asia. As shown in Fig. 7, the career trajectories on the map also increase a lot in the latest ten years. But the increase is not as obvious as in the last decade.

With the development of globalization, the distinguished AI scientists are more likely to move around the world. This increasing trend has become more apparent since the beginning of the new century. Although a rigid system may discourage native-born researchers from emigrating, openness is required by science. One take-away from a national policy perspective, two kinds of policies can be adopted: 1) encouraging young students to go abroad, and creating opportunities for them to return back; 2) the loose immigrant policy for distinguished AI scientist is the important carrier for the promotion of AI development in a country.

\subsection{Mobility vs. Creativity}

With the development of the market-oriented economy, the flow of scientific talents is appearing with increasing frequency. It is plausible, although hard to prove that the countries with openness to foreign scientists have benefited from the mobility of scientists. We can catch a glimpse of the relationship between mobility and creativity through scientists' outcomes. Here, we analyze the relationship between mobility and creativity from two aspects, including 1) time interval between job-hopping and 2) mobility versus scientific outputs.

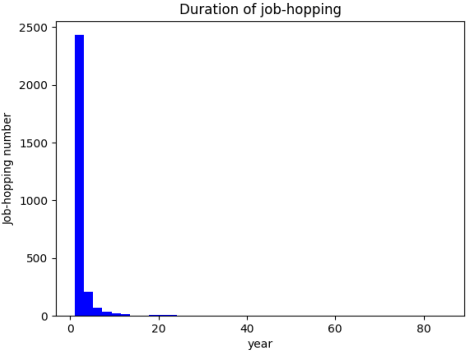

Fig. 8: Time interval between job- Fig. 9: Mobility vs. scientific outhopping.

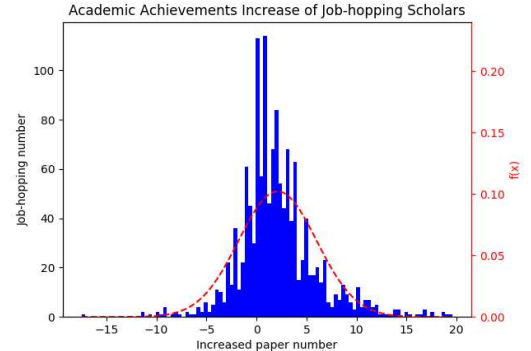

comes.

Q4.4: How often do AI scientists change their job positions?

The answer to this question is illustrated in Figure. 8. The distribution of the time interval between job-hopping is concentrated. The time interval is principally concentrated on 4 to 5 years. The average value of the time interval between job-hopping is 4.54 years. That is, the distinguished AI scholars will get a new job position in 4 to 5 years. The results break the usual cognitive 
structure. The time interval is shorter than people might think. Although it is difficult to obtain the detailed decision reasons underlying mobility, the results show high-frequency mobility of AI scientists.

Q4.5: What's the relationship between mobility and creativity?

We analyze all the job-hopping records of these AI scientists and obtain the fitted curve of the relation between scientific outcomes and job-hopping numbers. The curve is in a normal distribution, in which the mean and the standard deviation are 2.11 and 3.91 , respectively. We find that the publication number of every scholar will increase 2.55 in average after a job-hopping. It means that AI scientists are more productive in a job-hopping cycle than in the previous one. From another angle, the job positions of AI scientists will change when their scientific outcomes increase to a new level. The mobility of AI scientists has actually boosted their creativity. Although it is difficult to obtain detail reasons underlying mobility decisions of AI scientists, it is plausible to make a deduction that with the development of the AI technology, the flow of AI scientists are appearing with increasing frequency, and in turn, promote the development of AI technique further.

\section{The Evolution of Collaboration}

In this section, we emphasize the evolution of collaboration. The collaborative relationship between scientists are clearly reflected in their research publications. Here we take advantage of the fact that research findings of AI scientists are available online. We analyze all the research papers published by the distinguished AI scientists to reveal the collaboration relationship between these AI scientists.

\subsection{The Collaboration Distribution}

\section{Q5.1: What is the distribution of collaboration numbers?}

The number distribution of collaboration between the distinguished AI scientists is shown in Fig. 10, in which the left figure shows the number distribution, and the right figure shows the ratio distribution. A collaboration between scientists means that they have cooperated to publish an academic paper. As illustrated in the figure, there are: 1 ). 1324 scholars cooperate $0-9$ times, the corresponding ratio is $57.2 \% ; 2$ ). 630 scholars cooperate $10-19$ times, and the corresponding ratio is $27.2 \% ; 3$ ). 207 scholars cooperate $20-29$ times, and the corresponding ratio is $8.9 \% ; 4) .87$ scholars cooperate $30-39$ times, and the corresponding ratio is $3.8 \%$; 5). 41 scholars cooperate $40-49$ times, and the corresponding ratio is $1.8 \%$; 6 ). 25 scholars cooperate $50-59$ times, and the corresponding ratio is $1.1 \%$; 


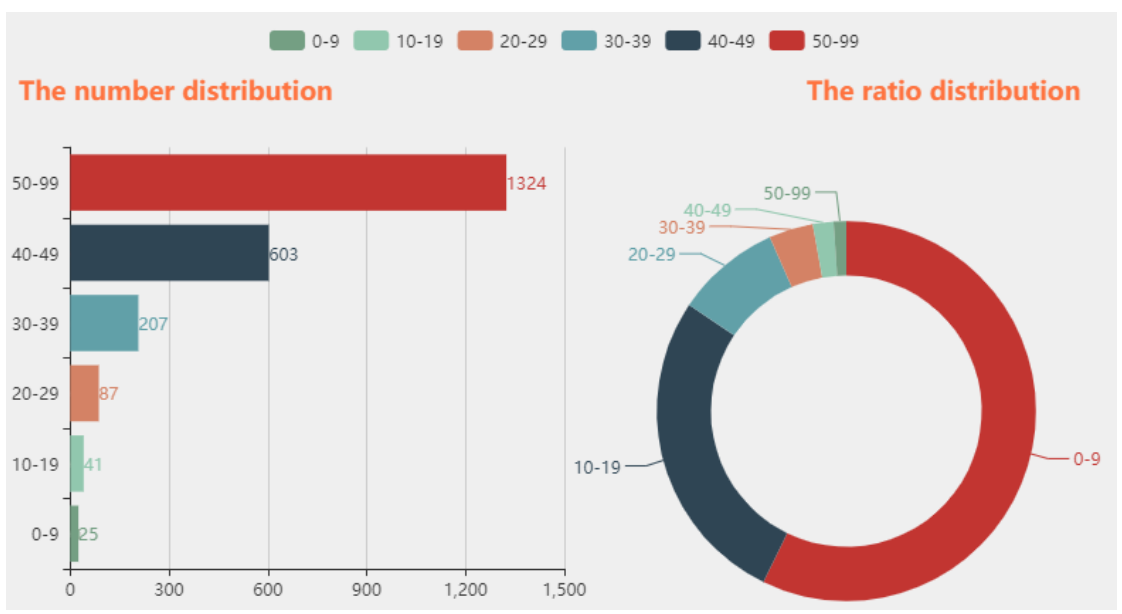

Fig. 10: The distribution of collaboration number.
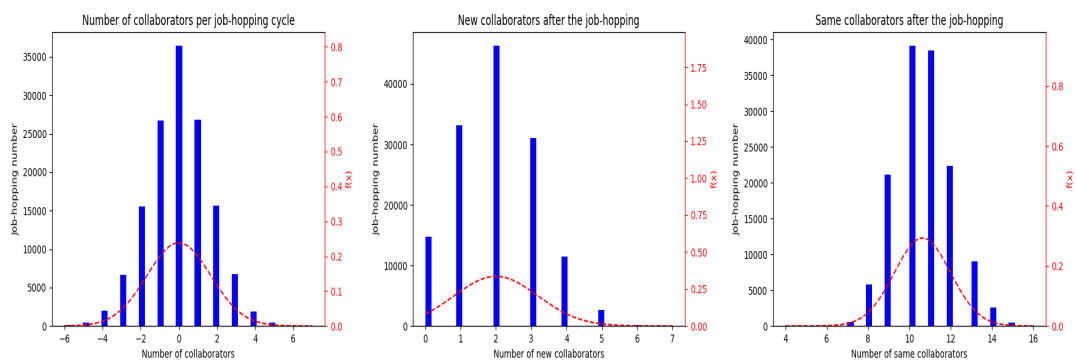

Fig. 11: Number of col- Fig. 12: New Collabo- Fig. 13: Remained collaborators. rators. laborators.

\subsection{The Collaboration Network}

Q5.2: what is the collaboration network between AI scientists?

From the publications of AI scientists, we construct a collaboration graph with collaboration relationships connecting pairs of authors that have coauthored at least on one paper. We analyze the collaboration network of distinguished AI scientists. Fig. 14 shows the collaboration relationship among all the distinguished AI scientists. The total number of analyzed AI scientist is 2433. There are 2314 nodes connected to the collaboration network. $95.11 \%$ distinguished AI scientists are interconnected with each other.

To study the most closely linked part in the collaboration network, we select the scholars, who cooperate with more than 10 distinguished AI scientists, 


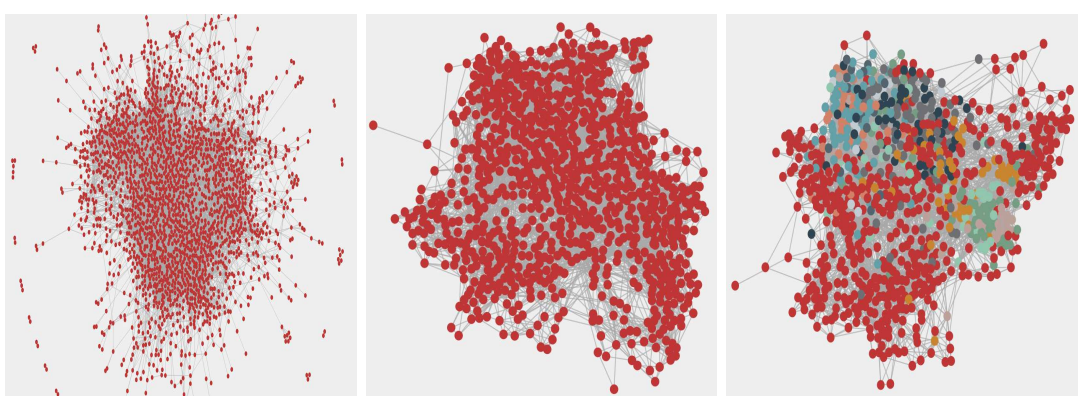

Fig. 14: All the distin- Fig. 15: Active AI Fig. 16: Colorized analguished AI scholars. scholars. ysis of active scholars.

as the active scholars. The collaboration network of active scholars is shown in Fig. 15. There are 890 active scholars. The number accounts for a large proportion of the collaboration network. With the development and popularity of the Internet, it is easier to work with collaborators at a distance. There are more collaborations between scientists than before [7].

To further analyze the collaboration situation, we colorize the nodes in the maximal connected subgraph. The top 20 AI scientists with the largest number of collaborators and their collaborators are colorized with the same color. The remaining scientists and their collaborators are labeled as red. We find that quite a number of nodes are colorized in Fig. 16. This means that these AI scientists are working in close collaboration with each other.

Especially, we analyze the cooperation network of Geoffrey E. Hinton, which is illustrated in Fig. 17. The point in Fig. 17 shows the academic influence of scholars (use h-index), and the value on the edge denotes the number of cooperation between scholars. The result shows that Geoffrey E. Hinton has 327 collaborations with 34 of the most influential scholars in his scholar career. The top 10 collaborators are shown in Table 5 , which counts the cooperation times (frequency) and the $\mathrm{H}$-index of the scholar.

Table 5: Top 10 collaborators of Geoffrey E. Hinton

\begin{tabular}{ccc}
\hline Name & h-index & frequency \\
\hline Ruslan Salakhutdinov & 67 & 27 \\
Richard Zemel & 52 & 20 \\
Zoubin Ghahramani & 91 & 18 \\
Max Welling & 65 & 17 \\
Ilya Sutskever & 35 & 17 \\
Andriy Mnih & 16 & 12 \\
George E. Dahl & 25 & 11 \\
Yee-Whye Teh & 52 & 10 \\
Abdel-Rahman Mohamed & 25 & 10 \\
Alex Krizhevsky & 10 & 10 \\
\hline
\end{tabular}




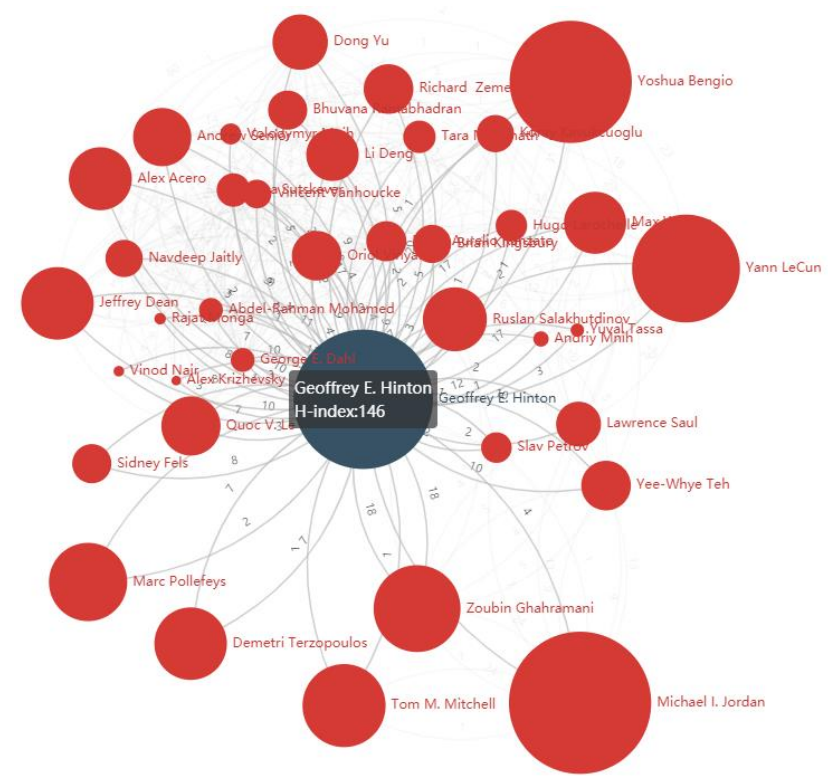

Fig. 17: Cooperpation Network of Geoffrey E. Hinton

\subsection{Collaboration vs. Mobility}

We take advantage of the fact that scientists publish research papers somewhat regularly, and the collaborative relationships between them are recorded in the publications. Here, we analyze how the collaboration pattern of AI scientists is changed along with their mobility.

Q5.3: How does the number of collaborators change?

We analyze all the job-hopping records of these AI scientists and obtain the fitted curve of the relation between the number of collaborators and jobhopping number, as shown in Figure 11. The curve is in a normal distribution, in which the mean and the standard deviation are 0 and 1.67, respectively. It means that the number of collaborators remains essentially unchanged, no matter whether the job position of AI scientists changes or not.

Q5.4: How many new collaborators have been increased?

We illustrate the relationship between the number of new collaborators and job-hopping numbers in Figure 12. The fitted curve is in a normal distribution, in which the mean and the standard deviation are 2 and 1.19, respectively. The number of new collaborators will increase 2 when the job position of AI scientists changes. As shown in the resulting figure, it is obvious that the average number of new collaborators will increase 2 when the job position of AI scientists changes.

Q5.5: How many collaborators will be retained?

We analyze how many collaborators will remain after job-hopping to answer this question - the relationship between the numbers of remained collab- 
orators and job-hopping number in shown Figure 13. The fitted curve is also in a normal distribution, in which the mean and the standard deviation are 10.63 and 1.36, respectively. The average number of remained collaborators is about 10 when the job position of AI scientists changes. Most of the AI scientists will maintain the relationship with each other even when their job positions have changed. Generally, the number of collaborators remains unchanged, and there are 2 new collaborators, and 10 old collaborators remained on average when the job position changes.

\section{Related Work}

Science of science, which is an emerging discipline wherein science is used to study the structure and evolution of science itself using large data sets, leads to a quantitative understanding of the genesis of scientific discovery, creativity, and practice [8]. Based on a transdisciplinary, SciSci studies the mechanisms underlying the doing of science, from the progress within a field to the factors on science evolution, aiming at accelerating scientific progress. The increasing availability of digitalized papers on academic and scientific online systems, such as AMiner [28], Google Scholar [30], and Microsoft Academic Services [24], offers unprecedented opportunities to explore the development of science. Identifying fundamental drivers of science and developing predictive models to capture its evolution are instrumental for successful science.

In the early research work, tremendous efforts have been devoted to the evaluation metrics for the quality and influence of scientific work, such as citation count [16], h-index [10], and impact factor [9]. Recognizing the fact that these scientific impact metric can only capture the past accomplishment, researchers try to predict the future trend of scientific work [1][32][22]. Research results reveal the regularity of scientific progress that a few research papers attract the vast majority of citations [2], long-distance interdisciplinarity leads to higher scientific impact [14][33]. In addition to the impact prediction, a lot of researches has been focused on the characterization of scientific impact, such as the universal citation distributions [19], the quantification of coauthor contributions [20], and the growth pattern of h-index [6]. Further, network science has been introduced into the analysis of scientific collaboration and citation, including the characteristics of citation networks [11][18][13], the structure of scientific collaboration networks [17][27], and so on.

Recent research efforts explore SciSci to unveil the evolution of science [26][3]. Wagner et al. [31] give a review of the literature to understand interdisciplinary scientific research. The investigation of the underlying trends in scientists' movements reveals that science shows the trend of increasing globalization [29]. Internationally mobile scholars show high impact across the globe, the circulation limitation of scholars will damage the entire scientific system [25]. Deville et al. [5] track the affiliation information of selected scientists to reconstruct their career trajectories. They find that career movement is not only temporally and spatially localized but also characterized by a high degree 
of stratification in the institutional ranking. Jia et al. [12] perform a large-scale analysis of publication records, which discovers a high degree of regularity underlying scientific research and individual careers. They present a quantitative analysis of macroscopic patterns governing research interest change and find that research interest change follows a reproducible pattern characterized by an exponential distribution. Cimini et al. [4] discuss the science of science policy by using bibliometric data over 18 years since 1996 collected from the SCImago website. They discuss the contribution of research funding and rate of international collaboration to research performance. They find significant differences among nations in terms of efficiency in turning (financial) input into bibliometrically measurable output and confirm that growth of international collaboration positively correlates with scientific success, with significant benefits brought by EU integration policies.

Two very recent related work examines the progress of Physics research and scientific development, respectively. Sinatra et al. [23] give a development analysis of physics-based on Web of Science data spanning more than 100 years. The results reveal the rapid growth and increasing multidisciplinarity of physics, as well its internal map of subdisciplines. Dong et al. [7] study the evolution of scientific development over the past century by presenting an anatomy of 89 million digitalized papers published between 1900 and 2015. Different from the work in [23], their study provides evolutionary and planetaryscale views of scientific development.

\section{Conclusions}

In this work, we present the evolution of AI from three aspects, including trend, mobility, and collaboration. Mathematics and theory related AI researches are more popular than applied-oriented AI researches in the early period, but vice versa in the following period. With the largest number of distinguished AI scientists, the USA is the most popular choice for scientists who tend to emigrate. The brain drain problem of distinguished AI scientists is continuing in developing countries. The increasing mobility of AI scientists, which has become apparent since the beginning of the new century, has actually boosted their creativity. The ties among AI elites are highly clustered in the collaboration network. Overall, our findings unveil the evolutionary patterns of AI, which benefit for more scientific AI policies.

Acknowledgements The work is supported by the National Natural Science Foundation of China (NSFC) under Grant No. 61806111, No. 61806109 and NSFC for Distinguished Young Scholar under Grant No. 61825602.

\section{References}

1. Acuna, D.E., Allesina, S., Kording, K.P.: Future impact: Predicting scientific success. Nature 489(7415), 201 (2012) 
2. Barabási, A.L., Song, C., Wang, D.: Publishing: Handful of papers dominates citation. Nature 491(7422), 40 (2012)

3. Bromham, L., Dinnage, R., Hua, X.: Interdisciplinary research has consistently lower funding success. Nature 534(7609), 684 (2016)

4. Cimini, G., Zaccaria, A., Gabrielli, A.: Investigating the interplay between fundamentals of national research systems: performance, investments and international collaborations. Journal of Informetrics 10(1), 200-211 (2016)

5. Deville, P., Wang, D., Sinatra, R., Song, C., Blondel, V.D., Barabási, A.L.: Career on the move: Geography, stratification, and scientific impact. Scientific reports 4, 4770 (2014)

6. Dong, Y., Johnson, R.A., Chawla, N.V.: Will this paper increase your h-index?: Scientific impact prediction. In: Proceedings of the eighth ACM international conference on web search and data mining, pp. 149-158. ACM (2015)

7. Dong, Y., Ma, H., Shen, Z., Wang, K.: A century of science: Globalization of scientific collaborations, citations, and innovations. In: Proceedings of the 23rd ACM SIGKDD International Conference on Knowledge Discovery and Data Mining, pp. 1437-1446. ACM (2017)

8. Fortunato, S., Bergstrom, C.T., Börner, K., Evans, J.A., Helbing, D., Milojević, S., et al.: Science of science. Science 359(6379) (2018)

9. Garfield, E.: Impact factors, and why they won't go away. Nature 411(6837), 522 (2001)

10. Hirsch, J.E.: An index to quantify an individual's scientific research output. Proceedings of the National academy of Sciences 102(46), 16569-16572 (2005)

11. Hunter, D., Smyth, P., Vu, D.Q., Asuncion, A.U.: Dynamic egocentric models for citation networks. In: Proceedings of the 28th International Conference on Machine Learning (ICML-11), pp. 857-864 (2011)

12. Jia, T., Wang, D., Szymanski, B.K.: Quantifying patterns of research-interest evolution. Nature Human Behaviour 1(4), 0078 (2017)

13. Kuhn, T., Perc, M., Helbing, D.: Inheritance patterns in citation networks reveal scientific memes. Physical Review X 4(4), 041036 (2014)

14. Larivière, V., Haustein, S., Börner, K.: Long-distance interdisciplinarity leads to higher scientific impact. Plos one 10(3) (2015)

15. Ma, Y., Uzzi, B.: Scientific prize network predicts who pushes the boundaries of science. Proceedings of the National Academy of Sciences 115(50), 12608-12615 (2018)

16. Margolis, J.: Citation indexing and evaluation of scientific papers. Science 155(3767), 1213-1219 (1967)

17. Newman, M.E.: The structure of scientific collaboration networks. Proceedings of the national academy of sciences 98(2), 404-409 (2001)

18. Pan, R.K., Kaski, K., Fortunato, S.: World citation and collaboration networks: uncovering the role of geography in science. Scientific reports 2, 902 (2012)

19. Radicchi, F., Fortunato, S., Castellano, C.: Universality of citation distributions: Toward an objective measure of scientific impact. Proceedings of the National Academy of Sciences 105(45), 17268-17272 (2008)

20. Sekercioglu, C.H.: Quantifying coauthor contributions. Science 322(5900), 371-371 (2008)

21. Shao, Z., Tang, J., Zhang, Y., Gao, B., Wang, Y.: Scholar trajectory: Visualizing the migration of talents (2018)

22. Shen, H.W., Wang, D., Song, C., Barabási, A.L.: Modeling and predicting popularity dynamics via reinforced poisson processes. In: AAAI, vol. 14, pp. 291-297 (2014)

23. Sinatra, R., Deville, P., Szell, M., Wang, D., Barabási, A.L.: A century of physics. Nature Physics 11(10), 791 (2015)

24. Sinha, A., Shen, Z., Song, Y., Ma, H., Eide, D., Hsu, B.j.P., Wang, K.: An overview of microsoft academic service (mas) and applications. In: Proceedings of the 24th international conference on world wide web, pp. 243-246. ACM (2015)

25. Sugimoto, C.R., Robinson-García, N., Murray, D.S., Yegros-Yegros, A., Costas, R., Larivière, V.: Scientists have most impact when they're free to move. Nature News $\mathbf{5 5 0}(7674), 29$ (2017)

26. Sun, X., Kaur, J., Milojević, S., Flammini, A., Menczer, F.: Social dynamics of science. Scientific reports 3, 1069 (2013) 
27. Tang, J., Wu, S., Sun, J., Su, H.: Cross-domain collaboration recommendation. In: Proceedings of the 18th ACM SIGKDD international conference on Knowledge discovery and data mining, pp. 1285-1293. ACM (2012)

28. Tang, J., Zhang, J., Yao, L., Li, J., Zhang, L., Su, Z.: Arnetminer: extraction and mining of academic social networks. In: Proceedings of the 14th ACM SIGKDD international conference on Knowledge discovery and data mining, pp. 990-998. ACM (2008)

29. Van Noorden, R.: Global mobility: Science on the move. Nature News 490(7420), 326 (2012)

30. Van Noorden, R.: Google scholar pioneer on search engine's future. Nature News (2014)

31. Wagner, C.S., Roessner, J.D., Bobb, K., Klein, J.T., Boyack, K.W., Keyton, J., Rafols, I., Börner, K.: Approaches to understanding and measuring interdisciplinary scientific research (idr): A review of the literature. Journal of informetrics 5(1), 14-26 (2011)

32. Wang, D., Song, C., Barabási, A.L.: Quantifying long-term scientific impact. Science 342(6154), 127-132 (2013)

33. Yegros-Yegros, A., Rafols, I., D'Este, P.: Does interdisciplinary research lead to higher citation impact? the different effect of proximal and distal interdisciplinarity. PloS one 10(8) (2015) 